\title{
Gastric emptying in patients with fundal gastritis and gastric cancer
}

\author{
M Tatsuta, H Iishi, S Okuda
}

\begin{abstract}
Gastric emptying was compared in patients with gastric cancers and fundal gastritis to determine its value in identifying patients at high risk of gastric cancer. Gastric emptying was measured by the acetaminophen absorption method, and the extent of fundal gastritis was determined by the endoscopic Congo red test. The results showed that gastric emptying was significantly slower in patients with severe fundal gastritis than in those without. Gastric emptying in patients with differentiated adenocarcinomas was significantly slower than in those with undifferentiated adenocarcinoma, its value being similar to that in patients with severe fundal gastritis. The Congo red test showed that the incidence of severe fundal gastritis was significantly greater in patients with differentiated adenocarcinomas than in those with undifferentiated cancers. These findings suggest that delayed gastric emptying may allow prolonged contact between dietary carcinogens and the stomach.
\end{abstract}

The past decade has witnessed an exponential growth in knowledge about gastric motility. Many disorders such as peptic ulcer, gastrooesophageal reflux, and non-ulcer dyspepsia are associated with delayed gastric emptying without evidence of structural gastric outlet obstruction.' We recently measured gastric emptying by the acetaminophen absorption method and found that its rate was significantly slower in patients with severe fundal gastritis than in those without this disorder. ${ }^{2}$ There have, however, been only a few reports on gastric emptying in patients with gastric cancers to date, ${ }^{3-6}$ and the results obtained have been conflicting. Because of this we measured gastric emptying in early gastric cancer and compared it with that in atrophic gastritis to determine its value as a parameter for identifying patients at high risk of gastric cancer.

Departments of Gastrointestinal Oncology and Gastroenterology, The Centre for Adult Diseases, Osaka, Japan M Tatsuta

$\mathrm{H}$ Iishi

S Okuda

Correspondence to:

Dr M Tatsuta, Department of Gastrointestinal Oncology,

The Centre for Adult Diseases,

Osaka, 3-3, Nakamichi

1-chome, Higashinari-ku,

1-chome, Higash
Osaka 537, Japan.

Accepted for publication

11 September 1989

\section{Patients and methods}

A total of 61 patients in two separate groups participated in this study. The first group consisted of 31 patients (20 men and 11 women whose mean age was $50 \cdot 3$ years (range 20-71 years)). These patients were suspected of having some type of gastric disease on the basis of radiological examination but were found to have no localised gastric lesions on fibreoptic endoscopy. Special tests were performed before the study to confirm that these patients did not have either pancreatohepatobiliary disease or pernicious anaemia. The second group comprised 30 patients with early gastric cancer (18 men and
12 women; mean age 52.9 years (range 42-75 years)). Informed consent was obtained from all patients and the study was carried out in accordance with the Declaration of Helsinki.

Gastric emptying was measured by the acetaminophen absorption method..$^{7-10}$ After overnight fasting the patients rapidly ingested $1.5 \mathrm{~g}$ of acetaminophen powder mixed with a semiliquid test meal. A commercially available diet (Okunos-A, $200 \mathrm{ml}$; Okuno Co, Tokyo, Japan) was used as the test meal. (Some $200 \mathrm{ml}$ of the diet contained $9.8 \mathrm{~g}$ protein, $5.2 \mathrm{~g}$ fat, $28.6 \mathrm{~g}$ carbohydrate, and $200 \mathrm{kcal}$.) Serum acetaminophen was determined by high performance liquid chromatography as described by Ameer et al." Gastric emptying was expressed as the serum acetaminophen value 45 minutes after ingestion of the test meal. ${ }^{27}$

The extent of fundal gastritis was judged by the endoscopic Congo red test. Details of the procedure for this test have been reported elsewhere. ${ }^{12}$ In brief, a fibreoptic endoscope was inserted into the stomach and a solution of $0.3 \%$ Congo red and $0.2 \mathrm{M}$ sodium bicarbonate was then sprayed over the whole surface of the stomach. An augmented dose $(5 \mu \mathrm{g} / \mathrm{kg})$ of tetragastrin (Tetragastrin-NS; Nissui Pharmaceutical Co, Tokyo, Japan) was injected intramuscularly and acid secretion was observed for the next five to 15 minutes. Areas where the Congo red turned blue-black because of acid secretion were designated 'discoloured' areas and those where the dye did not change colour were designated 'non-discoloured' areas.

Gastric biopsy specimens (average number per subject, 6.8 (range 4-9)) were obtained from discoloured and non-discoloured areas within $1 \mathrm{~cm}$ of the boundary between these two areas to examine histological features. They were fixed in neutralised $10 \%$ formalin, embedded in paraffin, and sectioned at $4 \mu \mathrm{m}$. Sections were stained with haematoxylin and eosin and examined without any knowledge of their source.

Following the classification of Whitehead et al..$^{13}$ the mucosa was distinguished as fundic or antral. When only intestinal metaplasia was seen, the specimens were regarded as unclassified. Gastritic changes were classified histologically as (1) superficial gastritis; (2) atrophic gastritis; and (3) gastric atrophy. ${ }^{14}$

The degree of fundal gastritis was classified on the basis of the extent of the non-discoloured areas in the gastric body as follows ${ }^{15} 16$ : (1) none the discoloured area was so extensive that nondiscoloured areas were scarcely detectable in the gastric body; (2) slight - non-discoloured areas extended slightly proximally from the lower corpus or were detectable only around the cardiac orifice; (3) moderate - non-discoloured areas were present along the lesser curvature 
from the angulus to the cardia; and (4) severe discoloured areas were limited to part of the greater curvature, or no acid secretion was detectable.

The final assessment of the spread and histological type of the gastric cancer was determined by histological examination of specimens obtained at gastrectomy. Early gastric cancers were defined as those confined to the mucosa or submucosa that did not affect the muscularis propria. ${ }^{17}$ Gastric cancers were histologically classified as either differentiated or undifferentiated types. The former had a tubular or papillary structure, whereas the latter showed loss of the glandular arrangement. A variable number of the cells were mucus secreting and sometimes they had a classic 'signet ring' appearance.

Data are given as mean (SE). Results were analysed by the $\chi^{2}$ test with Yates's correction ${ }^{18}$ or by Wilcoxon's rank sum test. ${ }^{19} \mathrm{~A}$ calculated $p$ value of less than 0.05 was regarded as significant.

\section{Results}

\section{HISTOLOGICAL FINDINGS IN DISCOLOURED AND NON-DISCOLOURED AREAS}

As shown in Table I, all 95 specimens collected from discoloured areas were found to consist of normal fundic mucosa. No fundal gastritis or antral mucosa was seen in these areas. Nondiscoloured areas, however, represented either antral mucosa or fundic mucosa altered by atrophic gastritis or gastric atrophy. Among the 217 specimens collected from non-discoloured areas in the gastric body, atrophic gastritis was found in $\mathbf{1 2 5}$ and gastric atrophy in $\mathbf{9 2}$. Therefore, the main histological feature of the nondiscoloured areas of the gastric body was fundal gastritis.

\section{GASTRIC EMPTYING IN FUNDAL GASTRITIS AND GASTRIC CANCER}

The data on gastric emptying in patients with fundal gastritis and in those with gastric cancer are summarised in Table II. The serum acetaminophen value was significantly lower in patients with severe fundal gastritis than in those with little, moderate, or no fundal gastritis. Table II also shows that the serum acetaminophen value was significantly lower in patients with differentiated adenocarcinoma of the stomach than in those with undifferentiated adenocarcinoma. The acetaminophen values in patients with differentiated adenocarcinoma and in patients with severe fundal gastritis were approximately equal.

CLINICAL AND HISTOPATHOLOGICAL FEATURES IN RELATION TO THE HISTOLOGICAL TYPES OF GASTRIC CANCER

The data on clinical and histopathological features in patients with differentiated and undifferentiated adenocarcinomas are summarised in Table III. The patients in the two groups did not differ significantly with regard to
TABLE I Histological findings in biopsy specimens from discoloured and non-discoloured gastric areas

\begin{tabular}{llc}
\hline Histological findings & $\begin{array}{l}\text { Discoloured } \\
\text { areas }(\%)\end{array}$ & $\begin{array}{l}\text { Non-discoloured } \\
\text { areas }(\%)\end{array}$ \\
\hline Fundic mucosa: & & \\
$\quad$ Normal & $95(100)^{\star}$ & $0(0)$ \\
Superficial gastritis & $0(0)$ & $0(0)$ \\
Atrophic gastritis & $0(0)$ & $125(39)$ \\
Gastric atrophy & $0(0)$ & $92(29)$ \\
Antral mucosa: & $0(0)$ & $0(0)$ \\
$\quad$ Normal & $0(0)$ & $0(0)$ \\
Superficial gastritis & $0(0)$ & $25(8)$ \\
Atrophic gastritis & $0(0)$ & $20(6)$ \\
Gastric atrophy & & $59(18)$ \\
Unclassified: & $0(0)$ & $321(100)$ \\
Gastric atrophy & $95(100)$ & \\
Total &
\end{tabular}

^Numbers of biopsy specimens examined.

sex distribution, age, location of gastric cancer, or extent of gastric cancer, although undifferentiated adenocarcinoma tended to be more frequently seen in women and younger patients. Table III also shows a significant correlation between the histological type of early gastric cancers and the extent of fundal gastritis. Differentiated adenocarcinomas were usually associated with severe fundal gastritis whereas undifferentiated cancers were associated with less severe fundal gastritis.

\section{Discussion}

Many methodological advances in the study of gastric emptying have taken place during the

TABLE II Gastric emptying in patients with fundal gastritis and gastric cancer

\begin{tabular}{lrl}
\hline Groups & & $\begin{array}{l}\text { Gastric emptying (serum } \\
\text { acetaminophen, } \mu g / m l\end{array}$ \\
\hline Fundal gastritis: & No & $($ mean $(S E))$ \\
$\quad$ None-slight & 11 & $7 \cdot 2(0 \cdot 6)$ \\
$\quad$ Moderate & 14 & $6 \cdot 4(0 \cdot 4)$ \\
$\quad$ Severe & 6 & $3 \cdot 9(0 \cdot 4)^{\star}$ \\
Early gastric cancer: & 15 & $3 \cdot 3(0 \cdot 3) \dagger$ \\
$\quad$ Differentiated adenocarcinoma & 15 & $6 \cdot 4(0 \cdot 5)$ \\
$\quad$ Undifferentiated adenocarcinoma & 15 & \\
\hline
\end{tabular}

$\star$ Significantly different from values for patients with little or no and for those with moderate fundal gastritis at $p<0.01$ (Wilcoxon's test).

†Significantly different from values for patients with undifferentiated adenocarcinoma and for those with little or no fundal gastritis, both at $\mathrm{p}<0.01$ (Wilcoxon's test).

TABLE III Clinical and histopathological data of patients with differentiated and undifferentiated adenocarcinoma of the stomach

\begin{tabular}{lll}
\hline Data & $\begin{array}{l}\text { Differentiated } \\
\text { adenocarcinoma }\end{array}$ & $\begin{array}{c}\text { Undifferentiated } \\
\text { adenocarcinoma }\end{array}$ \\
\hline No & 15 & 15 \\
Men:women & $10: 5$ & $8: 7$ \\
Age (yrs)(mean (SE)) & $59 \cdot 2(3 \cdot 1)$ & $53 \cdot 2(4 \cdot 2)$ \\
Location of gastric cancer & & \\
$\quad$ (no (\%)): & $1(7)$ & $0(0)$ \\
$\quad$ Upper corpus & $3(20)$ & $3(20)$ \\
Middle corpus & $3(20)$ & $3(20)$ \\
Lower corpus & $5(33)$ & $5(33)$ \\
Angulus & $3(20)$ & $4(27)$ \\
Antrum & $11(73)$ & $10(67)$ \\
Extent of gastric cancer: & $4(27)$ & $3(20)$ \\
$\quad<5 \mathrm{~cm}^{2}$ & $0(0)$ & $2(13)$ \\
$5-10 \mathrm{~cm}^{2}$ & $5(33)$ & $13(87)$ \\
$>10 \mathrm{~cm}^{2}$ & $10(67)^{\star}$ & $2(13)$ \\
Extent of fundal gastritis: & & \\
$\quad$ None-moderate & & \\
Severe & &
\end{tabular}

*Significantly different from the value for patients with undifferentiated adenocarcinomas at $p<0.01$ ( $\chi^{2}$ test with Yates's correction). 
past 15 years.' ${ }^{1}$ The acetaminophen absorption method is a reliable and relatively simple test to evaluate gastric emptying. ${ }^{70}$ Heading et $a l^{20}$ reported a significant correlation between the half life of gastric emptying, as measured by a sequential scintiscanning technique using a test meal plus radioisotope, and the serum acetaminophen values 30 and 60 minutes after drug ingestion. Similarly, Harrasawa et $\mathrm{al}^{7}$ reported a significant correlation between results obtained by isotopic studies and by this method.

In the present work we found noticeable delay in gastric emptying in patients with differentiated adenocarcinomas of the stomach but not those with undifferentiated adenocarcinomas. Nomiyama ${ }^{6}$ found, by use of the acetaminophen absorption method, that gastric emptying in patients with early gastric cancer was rather rapid compared with emptying in healthy subjects. Miwa et al, however, ${ }^{5}$ measured gastric emptying using an intubation technique and found that it was significantly slower in patients with early gastric cancer than in those with healed gastric ulcers. These differences may be caused by differences in the methods of gastric emptying measurement and in the test meals used.

Our present work has reconfirmed our previous results showing that gastric emptying was noticeably delayed in patients with severe fundal gastritis compared with those without this disorder. ${ }^{2}$ It was also found that the serum acetaminophen value in patients with differentiated adenocarcinomas of the stomach was similar to that in patients with severe fundal gastritis. The delay in gastric emptying in gastric cancer and atrophic gastritis leads to further speculation on the relation between these two conditions. We examined the role of fundal gastritis as a high risk factor by studying operative and necropsy findings and follow up data from patients with benign gastric disease on record at the Osaka Cancer Registry. We found that the risk of developing gastric cancer was significantly increased in patients with severe (but not moderate or slight) fundal gastritis (Tatsuta et al, submitted for publication). The antral portion of the inflamed stomach contains nitrite and is capable of nitrosating amines delivered to it by either the oral or the parenteral route. Oral administration of nitroso compounds causes inflammation, healing, atrophy, and intestinal metaplasia. Moreover, once an inflammatory process has been established it may become self perpetuating by means of the continuing endogenous production of nitroso compounds. ${ }^{21}$
Sommers ${ }^{3}$ has suggested that gastric cancers tend to develop at sites of prolonged contact between a carcinogen and abnormal gastric mucosa. In the present work we found that differentiated adenocarcinomas, but not undifferentiated adenocarcinomas, were associated with severe fundal gastritis. Atrophic gastritis may have a role in gastric cancer partly because of the associated delay in gastric emptying, which allows prolonged contact between dietary carcinogens and the stomach, and partly because of an inherent susceptibility of the gastric mucosa.

1 Minami H, McCallum RW. The physiology and pathophysiology of gastric emptying in humans. Gastroenterology physiology of gastric

2 Tatsuta M, Iishi H, Okuda S. Gastric emptying and gastrointestinal symptoms in patients with atrophic gastritis, and the effect of domperidone. Scand $\mathcal{F}$ Gastroenterol 1989; 24: 251-6.

3 Sommers SC. Constitutional aspects of gastric carcinoma. Arch Pathol 1958; 66: 487-93.

4 Davies WT, Kirkpatrick JR, Owen GM, Shields R. Gastric emptying in atrophic gastritis and carcinoma of the stomach. Scand F Gastroenterol 1971; 6: 297-301.

5 Miwa T, Tani N, Suzuki S, Fukutomi H, Sakita T. Gastric emptying in peptic ulcer and early gastric cancer. Naika 1976; 37: 643-7.

6 Nomiyama T. Gastric emptying in gastric cancer. In: Tsuneoka K, Miwa T, Sekiguchi T, eds. Gastroduodenal motor disorder. Tokyo: Bunkodo, 1985: 178-81.

7 Harasawa S, Tani T, Suzuki S, et al. Gastric emptying in normal subjects and patients with peptic ulcer. A study using the acetaminophen method. Gastroenterol f pn 1979; 14: $1-10$.

8 Kawamoto H, Yamamura H, Tatsuta M, Okuda S. Effect of glucagon on gastric motility examined by the acetaminophen absorption method and the endoscopic procedure. Arzmeimittelforschung 1985; 9: 1475-7

9 Clements JA, Heading RC, Nimmow WS, Prescott LF. Kinetics of acetaminophen absorption and gastric emptying in man. Clin Pharmacol Ther 1978; 24: 420-31.

10 Holt S, Heading RC, Clements JA, Tothill P, Prescott LF. Acetaminophen absorption and metabolism in celiac disease and Crohn's disease. Clin Pharmacol Ther 1981; 30: 232-8.

11 Ameer B, Greenblatt DJ, Divoll M, Abernethy DR, Shargel L. High-performance liquid chromatographic determination of acetaminophen in plasma: single-dose pharmacotion of acetaminophen in plasma: single-dose

12 Tatsuta M, Okuda S, Tamura H, Taniguchi H. Endoscopic diagnosis of early gastric cancer by the endoscopic Congo diagnosis of early gastric cancer by the endoscopic
red-methylene blue test. Cancer 1982; 50: 2956-60.

13 Whitehead S, Truelove AC, Gear MW. The histological diagnosis of chronic gastritis in fiberoptic gastroscope biopsy specimens. F Clin Pathol 1972; 25: 1-11.

14 Vilardell F. Chronic gastric disease and suction biopsy. Part 1: chronic gastritis. In: Bockus HI, ed. Gastroenterology. Vol 1. Philadelphia: WB Saunders, 1979.

15 Tatsuta $M$, Iishi $H$, Okuda S. Location of peptic ulcers in relation to antral and fundal gastritis by chromoendoscopic follow-up examinations. Dig Dis Sci 1986; 31: 7-11.

16 Tatsuta $M$, Okuda $S$, Taniguchi $H$, Tamura $H$. Gross and histological types of early gastric carcinomas in relation to the acid-secreting area. Cancer 1979; 43: 317-21.

17 Murakami T. Pathomorphological diagnosis. In: Murakami T, ed. Gann monograph on cancer research. Vol 11. Tokyo: University of Tokyo Press, 1971: 53-5.

18 Siegel S. Nonparametric statistics for the behavioral sciences. New York: McGraw-Hill, 1956.

19 Snedecor GW, Cochran WG. Statistical methods. Ames, IA: Iowa State University Press, 1967.

20 Heading R, Nimmo J, Prescott LF, Tothill P. The dependence of paracetamol absorption on the rate of gastric emptying. Brf Pharmacol 1973; 47: 415-21.

21 Stemmermann GN, Mower H. Gastritis, nitrosamines, and gastric cancer. $\mathcal{F}$ Clin Gastroenterol 1981; 3 (suppl 2): 23-7. 